\title{
SINGLE FAULT DETECTION AND DIAGNOSIS TECHNIQUE FOR DIGITAL MICRO-FLUIDIC BASED BIOCHIPS
}

\author{
Sagarika Chowdhury ${ }^{1}$, Sumitava Roy ${ }^{2}$, Sourav Saha ${ }^{2}$ and Arijit Saha ${ }^{2}$ \\ ${ }^{1}$ Assistant Professor, Computer Science \& Engineering Department, \\ Narula Institute of Technology, Kolkata, India \\ sag_saha2004@yahoo.co.in \\ ${ }^{2}$ Final Year Student, Computer Science \& Engineering Department, \\ Narula Institute of Technology, Kolkata, India \\ sumitava_royayahoo.com \\ qsourav@gmail.com \\ arijits1990@gmail.com
}

\begin{abstract}
This paper presents an integrated offline testing of Single-Fault detection technique for themicro-fluidic based biochips and also diagnosis single defects in order to achieve higherthroughput and less time complexity of the execution process. In the field operation, this is to be used to increase chip dependability and reusability of the biochips. In this paper, a pipelined technique is presented to traverse all the edges in much more less time in comparison with some previous techniques.
\end{abstract}

\section{KEYWORDS}

Biochip, Droplet, Fault detection \&diagnosis, Graph Dual \& Set theory, Pipeline

\section{INTRODUCTION}

Nowadays, the Digital Micro-fluidic based Biochip technologies are used either in development or concerning mercantile [1]-[3]. The composite system is also known as lab-on-a-chip. Such devices facilitate molecular biological procedures [15]. Rectification or Faultlessness, Constancy, Reusability, Sensitivity and Dependability are the important epithet of Micro-fluidic Biochips. These chips have to be examined both after the manufacturing and during the application.

A new advancing creation of droplet based micro fluidic device is also known as the "Digital Micro-fluidic Biochip" which was induced to imitate the electro wetting-on-dielectric principle [4][6]. The significant factor is the miniature size of these devices sustain to the shorter fault detection time, high throughput, lightening the energy in biological field operations [14].

The micro-fluidic biochips are categorized for the revelation of faults [7]-[10]. The benefit is to manipulate liquids as discrete droplets [3].

Micro-fluidic biochips have been characterized for detection of faults [1]-[3]. The test planning problem was formulated in terms of Euler circuit in [9]-[10]. Fei Su et. al. [16] has proposed defect tolerance based on graceful degradation and dynamic reconfiguration. In [17], a network flow based routing algorithm was proposed for the droplet routing problem on biochips. A novel parallel-scan like testing technique has been proposed in [12], where the total time slot covering the entire graph dual was considerably less. 
This paper introduces the disclosure of an efficient technique for diagnosis a single fault in a Micro-Fluidic Biochip having an optimum time complexity.

\section{Preliminaries}

\subsection{Component of a micro-fluidic array}

In digital micro-fluidic biochips, the liquids are divided into discrete and distinctly controllable manipulated microliter-nanolitre (the volume of the droplets) droplets, traverse on a two dimensional array, based on the principle of electro wetting-on-dielectric (EWOD) $[1,3,9]$.

The fundamental unit cell of a EWOD based digital micro-fluidic biochip composed of two parallel glass plates, as shown in Figure. 1 [9]. The top plate is implicated with a continuous ground electrode and the bottom plate holds a patterned array of electrodes. They are formed by Indium Tin Oxide and are regulated individually. A dielectric layer coated with hydrophobic film of Teflon AF is added to the plates to extenuate the wet ability of the surface and to enhance the capacitance between the droplets and the electrode [3].

Afiller medium like silicone oil is used in between the plates, where the droplets move. When an electric control voltage is applied to the electrode adjacent to the droplet, the electrode under the droplet is deactivated and moves the droplet to the active electrode because of the EWOD effect [13].

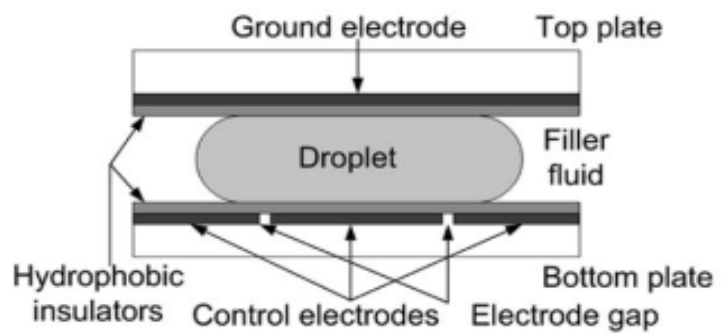

(a)

Figure 1.Structure of digital micro-fluidic biochip (Basic unit cell used in an EWOD-based digital

\subsection{Defect Characterization}

micro-fluidic biochip)

There are mainly two types of faults in digital micro-fluidic systems, catastrophic andparametric, as described in [7]. Catastrophic fault causes complete breakdown of the system, whereas parametric fault degrades system performance.

To detect a fault, we need to pass a droplet through the micro-fluidic array, so that it can traverse each and every cell and reach towards the sink. If there is any kind of fault within the microarray, the droplet will get stuck over there.

\subsection{Graph Theoretic Formulation}

Let us represent the entire micro-fluidic array as an mxn matrix where $\mathrm{C}_{\mathrm{i}, \mathrm{j}}$ denotes the cell at the $(i, j)$ position where $\mathrm{i}=1$ to $\mathrm{m}$ and $\mathrm{j}=1$ to $n$. The source and the sink, i.e., the positions outside the array from where the droplet enters the array and exits the array respectively, are designated by the cells adjacent to them.

Example 1.Figure. 2 shows a $3 \times 4$ micro-fluidic array with the source at $(1,1)$ and sink at the $(3$, 4) position. 


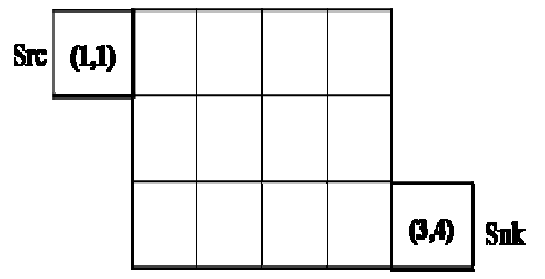

Figure 2.A 3x4 micro-fluidic array with source at $(1,1)$, sink at $(3,4)$

The source and sink are actually outside the array. The cell $(1,1)$ indicates the cell from which the droplet starts from the source. Similarly, cell $(3,4)$ is the last cell before the droplet leaves the array to the sink.

$$
1 .
$$

Let $<\mathrm{S}_{1}, \mathrm{~T}_{1}>,<\mathrm{S}_{2}, \mathrm{~T}_{2}>\ldots \ldots<\mathrm{S}_{\mathrm{k}}, \mathrm{T}_{\mathrm{k}}>$ be a sequence of droplet movements from source to sink, such that together they cover the entire array (i.e. all cells and internal boundaries) where $S_{\mathrm{i}}$ denotes a segment (cell) through which the droplet traverse at time $T_{i}$ towards sink. The complete traversal by a droplet from $<\mathrm{S}_{1}, \mathrm{~T}_{1}>$ to $<\mathrm{S}_{\mathrm{k}}, \mathrm{T}_{\mathrm{k}}>$ is referred to as a pass. Therefore, total time taken by a droplet to complete a pass is $\mathrm{T}_{1}+\mathrm{T}_{2}+\ldots \ldots+\mathrm{T}_{\mathrm{k}}$. If during its pass, the droplet does not reach the sink even after its time period, we conclude that the droplet has stuck in the array, and the biochip is faulty. Thus the fault detection problem is finding a sequence of passes, such that all cells and all internal boundaries of the micro-fluidic array are traversed by the droplets at least once, and the total is minimized. To diagnose the exact faulty point, now we need to check out the predefined look-up table having for which faulty node which droplets cannot reach the sink.

Definition 1.Graph Dual: Let $\mathrm{X}_{\mathrm{m}, \mathrm{n}}$ be an mxn micro-fluidic array. Then the dual of $\mathrm{Xm}, \mathrm{n}$ is a graph $\mathrm{G}=(\mathrm{V}, \mathrm{E})$ where each node $\mathrm{v} \in \mathrm{V}$ corresponds to a cell in the array and an edge $\mathrm{e}_{\mathrm{i}, \mathrm{j}}$ connecting the nodes $v_{i}$ and $v_{j}$ represent the boundary between the cells corresponding to the nodes $v_{i}$ and $\mathrm{v}_{\mathrm{j}}$ respectively, as described in [18].

Example 2.Figure 3. (a), (b) show a $4 \times 3$ micro-fluidic array, $\mathrm{X}_{4 \times 3}$ and its corresponding graph dual, $\mathrm{G}_{4 \times 3}$ respectively. The dotted lines represent the boundaries between the cells. They map to the edges of the Figure 3 (b). Each cell in Figure 3 (a). denotes a vertex in Figure 3 (b).
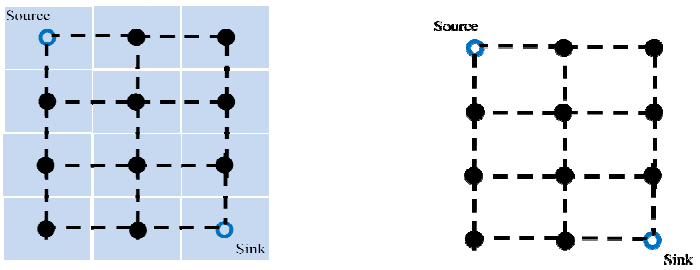

Figure 3.A 4x3 micro-fluidic array (left) and its graph dual (right)

The nodes of a graph dual are denoted as $\mathrm{v}_{\mathrm{ij}}$, where $\mathrm{v}_{\mathrm{ij}}$ corresponds to the cell $(\mathrm{i}, \mathrm{j})$. Now, the optimal fault detection problem for an mxn micro-fluidic biochip can be restated in terms of its graph dual as a Source to Sink Array Traversal Problem.

Given the graph dual $\mathrm{G}_{\mathrm{m} \times n}$ of a rectangular micro-fluidic biochip $\mathrm{X}_{\mathrm{m} \times \mathrm{n}}$ and the positions of the source $(i, j)$ and sink $(k, 1)$, to find a sequence of movements $\left.\left\langle S_{1}, T_{1}\right\rangle,\left\langle S_{2}, T_{2}\right\rangle, \ldots .,<S_{k}, T_{k}\right\rangle$ where $S_{\mathrm{i}}$ denotes a segment (cell) through which the droplet traverse at time $T_{\mathrm{i}}$ towards sink such that all the droplets visit each node and edge at least once altogether and the total time is minimized. 


\section{Proposed TechniQue}

The proposed technique takes a greedy approach to solve the Source to Sink Array Traversal Problem. It covers all the edges, as well as all the vertices, and in very less time slots. The coverage of the nodes and edges during a pass is achieved through certain movements.

From the concept of the set theory, we know that any set can be divided into its subsets and applying this technique, the single defect simulation can be solved in a very short time. The technique is being illustrated as follows:

\subsection{Movement Patterns}

Each traversal from the source to the sink node is mainly based on the Down-Left-Down-Right (DLDR) movement. Let $\mathrm{V}_{\mathrm{ij}}$ be the current node during a traversal. Then the DLDR movement pattern is defined as follows:

a)

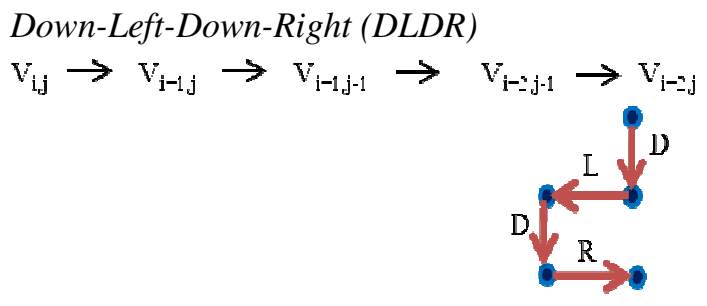

Figure 4.Down-Left-Down-Right movement pattern

\subsection{Strategy}

Let us locate four fixed nodes as the 'base' nodes from where the journey with the prescribed movement pattern begins. For the graph dual $G_{m x n}$, these base nodes are $B_{1}=V_{1,1}, B_{2}=V_{1, n}, B_{3}$ $=\mathrm{V}_{\mathrm{m}, 1}$, and $\mathrm{B}_{4}=\mathrm{V}_{\mathrm{m}, \mathrm{n}}$. These four nodes are shown in Figure 5. for $\mathrm{G}_{\mathrm{mxn}}$. The procedure is described below for $\mathrm{G}_{\mathrm{mxn}}$, where, $\mathrm{m}, \mathrm{n}>=2$.

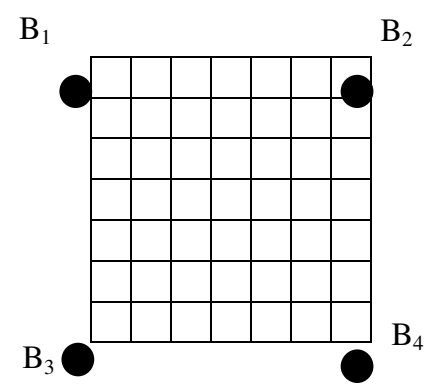

Figure 5. The four base nodes of a graph dual $\mathrm{G}_{\mathrm{mxn}}$

\subsection{The Complete Process}

In order to traverse all the edges of graph dual $G_{m x n}$, we will have all total $(m+n-2)$ passes, where $\mathrm{m}$ is the total number of rows and $\mathrm{n}$ is the total number of columns. All these passes will be followed by $(m+n-2)$ droplets in consecutive time quantum, e.g., Pass \#1 should be done in Timeslot \#1, and, in Time-slot \#2, thesecond pass should start its function, and in Time-slot \#3, Pass \#3 should commence traversing and so on. So, the droplets are in sequence and will traverse in parallel. The traversal path is described in Figure 6. (a)-(e). 
The Array-Traversal technique from a particular node, stated below:

\section{ProcedureArray-Traversal-Movement}

Step 1: Perform $D L D R$ movement pattern from the current node until last row is reached.

Step 2: If it is Sink node, stop there, otherwise from Current node arrive at the sink node, along the boundary considering the shortest path.

\section{End Procedure}

Example 3: Let us consider a graph dual $\mathrm{G}_{4 \times 3}$. Without loss of generality, let us suppose that $\mathrm{B}_{1}$ itself is the source. Figure 6. (a)-(e) show the path explored in pass \#1, Pass \#2, ..., Pass \# $(m+n-$ 2 ), where $m=4$ and $n=3$.

From the above example, it is clear that, total number of droplets, as well as passes will be (m-1) $+(n-1)=(m+n-2)$, whereas the existing algorithm [12], uses $(m+n)$ droplets.

\subsection{Analysis}

Table I shows the entire traversal procedure with respect to time and segment for a graph dual $\mathrm{G}_{4 \times 3}$ (Figure 6). Let us consider each node of this graph dual as $\mathrm{s}_{1}, \mathrm{~s}_{2} . \mathrm{s}_{3}, \ldots, \mathrm{s}_{12}$ respectively in row major order. In this table, each row represents each consecutive pass and it is clear from this table that no collision is occurred during the traversal of the droplets through the pipeline. It takes 11 unit times to complete the entire diagnosis procedure for a $4 \times 3$ micro-fluidic array.

TABLE I

TIMING AND COLLISION ANALYSIS

\begin{tabular}{|l|l|l|c|c|c|c|c|c|c|c|c|}
\hline $\mathbf{T}_{\mathbf{0}}$ & $\mathbf{T}_{\mathbf{1}}$ & $\mathbf{T}_{\mathbf{2}}$ & $\mathbf{T}_{\mathbf{3}}$ & $\mathbf{T}_{\mathbf{4}}$ & $\mathbf{T}_{\mathbf{5}}$ & $\mathbf{T}_{\mathbf{6}}$ & $\mathbf{T}_{\mathbf{7}}$ & $\mathbf{T}_{\mathbf{8}}$ & $\mathbf{T}_{\mathbf{9}}$ & $\mathbf{T}_{\mathbf{1 0}}$ & $\mathbf{T}_{\mathbf{1 1}}$ \\
\hline $\mathrm{S}_{1}$ & $\mathrm{~S}_{4}$ & $\mathrm{~S}_{7}$ & $\mathrm{~S}_{10}$ & $\mathrm{~S}_{11}$ & $\mathrm{~S}_{12}$ & & & & & & \\
\hline & $\mathrm{S}_{1}$ & $\mathrm{~S}_{4}$ & $\mathrm{~S}_{7}$ & $\mathrm{~S}_{8}$ & $\mathrm{~S}_{9}$ & $\mathrm{~S}_{12}$ & & & & & \\
\hline & & $\mathrm{S}_{1}$ & $\mathrm{~S}_{4}$ & $\mathrm{~S}_{5}$ & $\mathrm{~S}_{6}$ & $\mathrm{~S}_{9}$ & $\mathrm{~S}_{12}$ & & & & \\
\hline & & & $\mathrm{S}_{1}$ & $\mathrm{~S}_{2}$ & $\mathrm{~S}_{3}$ & $\mathrm{~S}_{6}$ & $\mathrm{~S}_{5}$ & $\mathrm{~S}_{8}$ & $\mathrm{~S}_{9}$ & $\mathrm{~S}_{12}$ & \\
\hline & & & & $\mathrm{S}_{1}$ & $\mathrm{~S}_{2}$ & $\mathrm{~S}_{5}$ & $\mathrm{~S}_{4}$ & $\mathrm{~S}_{7}$ & $\mathrm{~S}_{8}$ & $\mathrm{~S}_{11}$ & $\mathrm{~S}_{12}$ \\
\hline
\end{tabular}

Therefore, the total time required to complete the entire diagnosis process for an mxn matrix is as follows:

For even value of $m$ :

$(m+n-3)+2(m-1)+(n-2)=3 m+2 n-7$

For odd value of $m$ :

$(m+n-3)+2(m-1)+(n-1)=3 m+2 n-6$

For the simplicity, let us think, $m=n$. Therefore, the proposed algorithm will take $(5 n-C)$ unit time ( $C$ is a constant having value 6 or 7 as described before) which is less than the time, $8 \mathrm{~N}$ yields by [12]. Therefore, $\mathrm{T}(\mathrm{m}, \mathrm{n})=\mathrm{O}(\mathrm{m})$.

ProcedureSingle-Fault-Detection-and-Diagnosis-in- $G_{m \times n}$

Perform ( $\mathrm{m}+\mathrm{n}-2)$ passes, i.e., Pass \#1, Pass \#2 ,...., Pass \#(m-1), Pass \#m, Pass \#(m+1), ..... Pass $\#(m+n-2)$ in parallel, such that they start from the source node at $(m+n-2)$ consecutive time slots. 


\section{PassBegin}

\section{Pass \#1:}

Step 1: Locate the nearest base node $B_{i}$, for the given source.

Find the shortest path from the source to $B_{i}$ along the boundary of the graph dual $G_{m \times n}$.

Step 2: Go down up to $\mathrm{m}^{\text {th }}$ row from Base $\mathrm{B}_{\mathrm{i}}$, then go right up to last column $\left(\mathrm{n}^{\text {th }}\right.$ column).

If it is Sink node, stop there, otherwise from Current node arrive at the Sink node, along the boundary considering the shortest path.

\section{Pass \#2:}

Step1: Delay for one time slot.

Start from Source. Go to nearest Base $\mathrm{B}_{\mathrm{i}}$, found in previous pass.

Step2: Go down up to $(m-1)^{\text {th }}$ row from Base $B_{i}$, then go right up to the last column. If it is Sink node, stop there, otherwise from Current node arrive at the Sink node, along the boundary considering the shortest path.

\section{Pass \#(m-1):}

Step1: Delay for $(m-2)$ time slot.

Start from Source. Go to nearest Base $\mathrm{B}_{\mathrm{i}}$, found in previous pass.

Step2: Go down up to $2^{\text {nd }}$ row from Base $\mathrm{B}_{\mathrm{i}}$, then go right up to the last column.

If it is Sink node, stop there, otherwise from Current node arrive at the Sink node, along the boundary considering the shortest path.

\section{Pass \#m:}

Step1: Delay for $(m-1)$ time slot.

Start from Source. Go to nearest Base $\mathrm{B}_{\mathrm{i}}$, found in previous pass.

Step2: Go right up to the $\mathrm{n}^{\text {th }}$ column from base $\mathrm{B}_{\mathrm{i}}$.

Start Array-Traversal-Movement procedure.

\section{Pass \#(m+1):}

Step1: Delay for $m$ time slot.

Start from Source. Go to nearest Base $\mathrm{B}_{\mathrm{i}}$, found in previous pass.

Step2: Go right up to the (n-1) ${ }^{\text {th }}$ column from base $\mathrm{B}_{\mathrm{i}}$.

Start Array-Traversal-Movement procedure.

\section{Pass \#(m+n-2):}

Step1: Delay for $(m+n-1)$ time slot.

Start from Source. Go to nearest Base $\mathrm{B}_{\mathrm{i}}$, found in previous pass.

Step2: Go right up to the $2^{\text {nd }}$ column from base $B_{i}$.

Start Array-Traversal-Movement procedure.

\section{PassEnd}

Sense each and every droplet at the Sink at their expected instants. Have all of them reached the Sink at predefined time? If yes, then the chip is fault free. Otherwise the chip is faulty. Now using the concept of set theory, identify the faulty node. 


\section{End procedure}

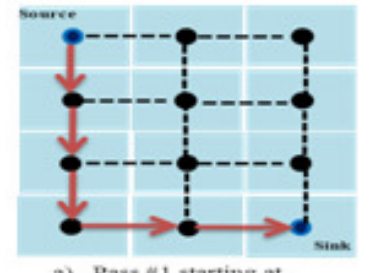

a) Pass $N 1$ starting at

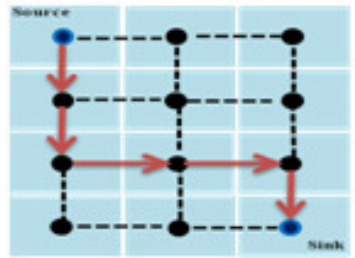

b) Pass 12 starting at

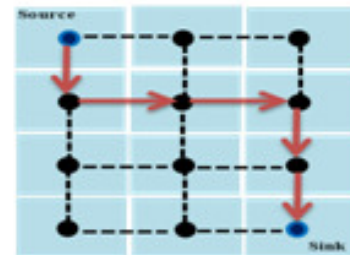

c) Pass 103 starting at Time-slot 13

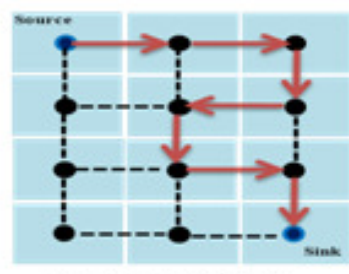

d) Pass 1.4 starting at

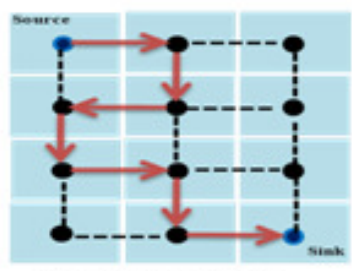

e) Pass 115 starting at

Figure 6.Traversal Procedure of Single-Fault-Diagnosis in $\mathrm{G}_{4 \times 3}$

\section{EXPERIMENTAL RESULTS}

Extensive experimentation was done with a large number of arrays varying from $4 \times 4$ to $40 \times 40$ electrodes. Table II, III and IV report three of the test cases. Table V reports the performance of the proposed technique with respect to the existing technique [12]. It is found that in all cases the proposed method yields better results in terms of lesser test time slices (no. of edges traversed) for the corresponding micro-fluidic biochip. Column 5 of table V shows the \% improvement in each case. This is defined as:

$$
\% \text { improvement }=\frac{\text { existing_time }- \text { proposed_time }}{\text { existing_time }} \times 100
$$

TABLE II

FAULTY NODE DIAGNOSIS

\begin{tabular}{|c|c|}
\hline $\begin{array}{c}\text { Defected } \\
\text { Node }\end{array}$ & $\begin{array}{c}\text { Only Droplets } \\
\text { Which Will Not } \\
\text { Reach Sink }\end{array}$ \\
\hline$(1,2)$ & Droplet 3,4 \\
\hline$(1,3)$ & Droplet 3 \\
\hline$(2,1)$ & Droplet $1,2,4$ \\
\hline$(2,2)$ & Droplet $2,3,4$ \\
\hline$(2,3)$ & Droplet 2,3 \\
\hline$(3,1)$ & Droplet 1,4 \\
\hline$(3,2)$ & Droplet $1,3,4$ \\
\hline
\end{tabular}

For a $3 \times 3$ micro-fluidic array 
TABLE III

FAULTY NODE DIAGNOSIS

\begin{tabular}{|c|c|}
\hline $\begin{array}{c}\text { Defected } \\
\text { Node }\end{array}$ & $\begin{array}{c}\text { Only Droplets } \\
\text { Which Will Not } \\
\text { Reach Sink }\end{array}$ \\
\hline$(1,2)$ & Droplet 4,5 \\
\hline$(1,3)$ & Droplet 4 \\
\hline$(2,1)$ & Droplet $1,2,3,5$ \\
\hline$(2,2)$ & Droplet $3,4,5$ \\
\hline$(2,3)$ & Droplet 3,4 \\
\hline$(3,1)$ & Droplet $1,2,5$ \\
\hline$(3,2)$ & Droplet $2,4,5$ \\
\hline$(3,3)$ & Droplet $2,3,4$ \\
\hline$(4,1)$ & Droplet 1 \\
\hline$(4,2)$ & Droplet 1,5 \\
\hline
\end{tabular}

For a 4x3 micro-fluidic array

TABLE IV

FAULTY NODE DIAGNOSIS

\begin{tabular}{|c|c|}
\hline $\begin{array}{c}\text { Defected } \\
\text { Node }\end{array}$ & $\begin{array}{c}\text { Only Droplets } \\
\text { Which Will Not } \\
\text { Reach Sink }\end{array}$ \\
\hline$(1,2)$ & Droplet 4,5,6 \\
\hline$(1,3)$ & Droplet 4,5 \\
\hline$(1,4)$ & Droplet 4 \\
\hline$(2,1)$ & Droplet $1,2,3,6$ \\
\hline$(2,2)$ & Droplet $3,5,6$ \\
\hline$(2,3)$ & Droplet $3,4,5$ \\
\hline$(2,4)$ & Droplet 3,4 \\
\hline$(3,1)$ & Droplet $1,2,6$ \\
\hline$(3,2)$ & Droplet $2,5,6$ \\
\hline$(3,3)$ & Droplet $2,4,5$ \\
\hline$(3,4)$ & Droplet $2,3,4$ \\
\hline$(4,1)$ & Droplet 1 \\
\hline$(4,2)$ & Droplet 1,6 \\
\hline$(4,3)$ & Droplet $1,5,6$ \\
\hline
\end{tabular}

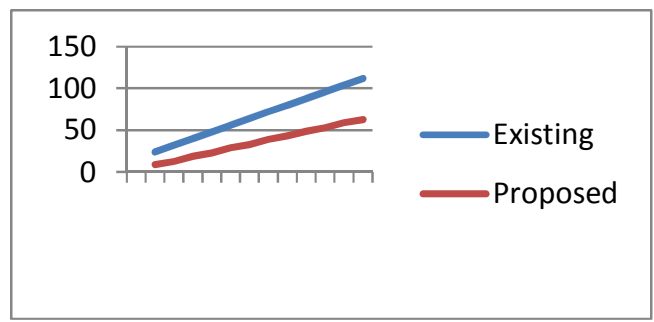

Figure 7. X Axis: Array Size and Y Axis: Unit Time 
TABLE V

PERFORMANCE Of THE PROPOSED TECHNIQUE

\begin{tabular}{|c|c|c|c|c|}
\hline $\begin{array}{c}\text { Sl. } \\
\text { No }\end{array}$ & Size & $\begin{array}{c}\text { Existing } \\
\text { Technique } \\
{[\mathbf{1 2}]}\end{array}$ & $\begin{array}{c}\text { Proposed } \\
\text { Technique }\end{array}$ & $\begin{array}{c}\text { \% } \\
\text { Improvement }\end{array}$ \\
\hline 1 & $3 \times 3$ & 24 & 9 & 62.50 \\
\hline 2 & $4 \times 4$ & 32 & 13 & 59.38 \\
\hline 3 & $5 \times 5$ & 40 & 19 & 52.50 \\
\hline 4 & $6 \times 6$ & 48 & 23 & 52.09 \\
\hline 5 & $7 \times 7$ & 56 & 29 & 48.22 \\
\hline 6 & $8 \times 8$ & 64 & 33 & 48.44 \\
\hline 7 & $9 \times 9$ & 72 & 39 & 45.84 \\
\hline 8 & $10 \times 10$ & 80 & 43 & 46.25 \\
\hline 9 & $11 \times 11$ & 88 & 49 & 44.32 \\
\hline 10 & $12 \times 12$ & 96 & 53 & 44.80 \\
\hline 11 & $13 \times 13$ & 104 & 59 & 43.27 \\
\hline 12 & $14 \times 14$ & 112 & 63 & 43.75 \\
\hline
\end{tabular}

\section{CONCLUSION}

An efficient single fault detection and diagnosis technique for digital micro-fluidic based Biochip has been proposed in this paper. Diagnosis technique in a digital micro-fluidic biochip is an essential key, because the subsequent performance of the biochip. Since the Dependability, Faultlessness are the important attribute of micro-fluidic based biochip, therefore the efficient diagnosis technique is extremely important factor. In this paper, the efficient defect-oriented testing, diagnosis methodology and significant experimental results have pointed out that the improvement of the test time over the existing proposed technique. Figure 7. shows the difference in the performance graph clearly. This is an offline proposed technique, so it is not possible to show any practical experimental result.

\section{REFERENCE}

[1] M. Pollack, et. al., "Electrowetting-based Actuation of Droplets for Integrated Microfluidics", Lab Chip, vol 2, pp. 96-101,2002.

[2] V. Srinivasan et al., "An Integrated Digital Microfluidic Lab-on-a-chip for Clinical diagnostics on Human Physiological Fluids", Lab Chip, pp.310-315, 2004.

[3] F. Su et. al.., "Architectural-level synthesis of Digital Microfluidics based Biochips", Proc. IEEE Int. Conf. on CAD,pp.223-228,2004.

[4] R. B. Fair et al., "Electrowetting-based on-chip sample processing for integrated microfluidics," in Proc. IEDM, 2003, pp. 32.5.1-32.5.4.

[5] E. Verpoorte and N. F. De Rooij, "Microfluidics meets MEMS," Proc. IEEE, vol. 91, no. 6, pp. 930953, Jun. 2003.

[6] J. Zeng and T.Korsmeyer, "Principles of droplet electrohydrodynamicsfor lab-on-a-chip," Lab on a Chip, vol. 4, pp. 265-277, 2004.

[7] F. Su ,S.Ozev and K. Chakrabarty, “ Testing of Droplet-based Microfluidic Systems”, Proc. IEEE Int. Test Conf.,pp.1192-1200,2003.

[8] F.Su, S. Ozev and K.Chakrabarty,'Test Planning and Test Resource Optimization for Droplet-Based Microfluidic Systems", Proc. IEEE Eur. Test Sym., pp. 72-77,2004.

[9] Fei Su, et. al.,"Testing and Diagnosis of Realistic Defects in Digital Microfluidic Biochip”, proc. 2007 Springer Science + Business Media.

[10] V. Srinivasan, et. al, "A Digital Microfluidic Biosensor for Multi analyteDetection”, Proc. IEEE MEMS Conference, pp. 327-33-, 2003., 
[11] S. K. Cho et al., "Creating, transporting, cutting, and merging liquid droplets by electrowetting-based actuation for digital microfluidic circuits," J. Microelectromech. Syst., vol. 12, pp. 70-80, 2003.

[12] Tao Xu and KrishnenduChakrabarty, “ Parallal Scan-Like Test and Multiple-Defect Diagnosis for Digital Microfluidic Biochips", IEEE TRANSACTIONS ON BIOMEDICAL CIRCUITS AND SYSTEMS, VOL. 1, NO. 2, JUNE 2007.

[13] R. B. M. Schasfoort et al., "Field-effect flow control for microfabricated fluidic networks," Science, vol. 286, pp. 942-945, 1999.

[14] "Two-dimensional digital microfluidic system by multi-layer printed circuit board," in Proc. IEEE MEMS, 2005, pp. 726-729.

[15] T. H. Schulte et al., "Microfluidic technologies in clinical diagnostics," ClinicaChim. Acta, vol. 321, pp. 1-10, 2002.

[16] Feisu, K. Chakrobarty, "Defect Tolerance Based on Graceful Degradation and Dynamic Reconfiguration for Digital Microfluidics-Based Biochips", IEEE TCAD V.25, No. 12, 2006.

[17] Tao Xu and K. Chakrabarty, "Functional Testing of Digital Microfluidic Biochip", ITC 2007.

[18] S. Saha, et. al. "An Efficient Single Fault Detection Technique for Micro-Fluidic Biochips", in Proc. IEEE Int. Conf on ACE, pp. 10-14, 2010.

\section{AUTHORS}

Sagarika Chowdhury received the M.Tech. degree in Computer Science and Application from University of Calcutta and stood 1st Class Third. She is currently an Assistant Professor of Computer Science \& Engineering Department at Narula Institute of Technology, Kolkata, India. Her current research projects include Fault Detection and Diagnosis Techniques for Micro-Fluidic Based Biochips.

Sumitava Royis pursuing the B.Tech. degree in Computer Science and Engineering from Narula Institute of Technology (presently in his final year). His research interests include Fault Detection and Diagnosis Techniques for Micro-Fluidic Based Biochips.

Sourav Sahais pursuing the B.Tech. degree in Computer Science and Engineering from Narula Institute of Technology (presently in his final year). His research interests include Fault Detection and Diagnosis Techniques for Micro-Fluidic Based Biochips.

Arijit Sahais pursuing the B.Tech. degree in Computer Science and Engineering from Narula Institute of Technology (presently in his final year). His research interests include Fault Detection and Diagnosis Techniques for Micro-Fluidic Based Biochips.
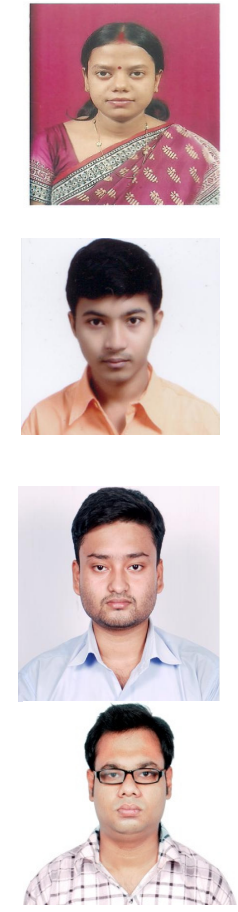